\title{
La nacionalización de los migrantes extranjeros: una capitalización social desde la participación asociativa y el compromiso cívico
}

\author{
The nationalization of foreign migrants: A social capitalization from \\ associative participation and civic engagement \\ José Manuel Gaete Fiscella* \\ Escuela de Trabajo Social, Universidad Academia de Humanismo Cristiano \\ Paola Ilabaca Baeza ${ }^{* * *}$ \\ Escuela de Psicología. Centro CIELO, Universidad Santo Tomás
}

Recibido: 8 de febrero de 2017. Aprobado: 25 de mayo de 2017.

\begin{abstract}
Resumen
El presente artículo indaga sobre la relevancia social de la nacionalización de los migrantes extranjeros en la sociedad chilena. Particularmente, exploramos hasta qué punto la naturalización de los inmigrantes, en tanto capitalización administrativa-legal dado el reconocimiento de derechos, también se asocia con una capitalización social más allá del ámbito formal, el nivel individual y, eventualmente, repercusiones beneficiosas, tanto para el grupo migrado como para la sociedad de acogida. Para esto, se analizaron las prácticas asociativas y sociales de migrantes nacionalizados y no nacionalizados a partir de una submuestra de la encuesta CASEN (2015). Los resultados obtenidos muestran que entre los migrantes nacionalizados, dado sus niveles y patrones de participación social, se presenta una mayor capitalización social respecto de los migrantes no nacionalizados.
\end{abstract}

Palabras claves: nacionalidad, migrantes, compromiso cívico.

\footnotetext{
Este artículo forma parte de los resultados de investigación del Proyecto Fondecyt postdoctorado № 3150449.

* Doctor en Sociología. Investigador postdoctoral FONDECYT. Académico de la Escuela de Trabajo Social, Universidad Academia Humanismo Cristiano. Correo electrónico: jgaetef@docentes.academia.cl

*** Doctora en Psicología. Investigadora Centro CIELO. Académica Escuela de Psicología, Universidad Santo Tomás. Correo electrónico: pilabaca@santotomas.cl
} 


\begin{abstract}
This article explores the social relevance of the nationalization of foreign migrants in Chilean society. In particular, we explore the extent to which the naturalization of immigrants, as an administrative-legal capitalization given the recognition of rights, is also associated with social capitalization that goes beyond the formal scope, the individual level and, eventually, beneficial aspects, both for the Group as well as for the host society. For this, the associative and social practices of nationalized and nonnationalized migrants were analyzed from a subsample of the CASEN survey (2015). The results obtained show that among the nationalized migrants, given their levels and patterns of social participation, there is a greater social capitalization with respect to nonnationalized migrants.
\end{abstract}

Key words: nationality, migrants, civic engagement.

\title{
Introducción
}

El fenómeno de la migración, principalmente desde los países vecinos a Chile, o también denominada sur-sur, ha sido un tema de vital interés en la discusión política nacional (Mora, 2008). Y es que desde mediados de los 90 se vive en el país un nuevo proceso migratorio que, y en comparación con los procesos vividos en el hemisferio norte, presenta particularidades y dimensiones propias (Mora, 2008). Se trata de un fenómeno que en Chile ha tomado un importante protagonismo a nivel académico, político y social. Esto ha supuesto que su estudio tome diversas aproximaciones, énfasis y objetivos; los que van desde la caracterización de los colectivos migrados hasta, aunque en menor medida, los enfoques que buscan evidenciar los aportes que los colectivos migrantes entregan a las sociedades de origen y de acogida.

En función de lo anterior, este trabajo aborda la cuestión planteada al intentar superar el foco de la problemática migrante (victimización y precarización) como elemento único y central sobre migración y migrantes ${ }^{1}$. Más bien se busca evaluar el aporte de los migrantes que, una vez nacionalizados, evidenciarían un mayor compromiso cívico con la sociedad

Por migrante se entiende a "los inmigrantes cuya vida cotidiana depende de múltiples y constantes interconexiones a través de fronteras internacionales y cuyas identidades públicas se configuran en relación con más de un Estado-nación" (Schiller, Basch y Blanc 1995, p. 48). 
de acogida, particularmente, bajo la forma de participación asociativa (capitalización social $^{2}$ ) en organizaciones nativas.

Para lo anterior, la investigación se centra en un migrante particular, nos referimos al migrante nacionalizado. Su particularidad se debe a varios elementos, el primero es que su emergencia en la sociedad de acogida responde a un proceso lento y construido en relación con la misma sociedad de acogida, en tanto el status de nacionalizado es sancionado por el propio aparato estatal y administrativo de la sociedad receptora. Por lo mismo, el migrante nacionalizado no es un migrante numeroso, y menos aún si se le compara con las oleadas recientes de migrantes que arriban a Chile, por lo que la atención que se le presta es menor y, con ello, las investigaciones sobre los mismos son más bien escasas. Así, se intenta exponer hasta qué punto la naturalización de los migrantes va más allá de una mera capitalización de derechos; más bien, y en suma con lo anterior, la nacionalización también se relacionaría con una capitalización social que supone aportes sociales y cívicos tanto para el migrante naturalizado como para la propia sociedad de acogida que lo naturaliza, en este caso, la sociedad chilena.

\section{La construcción y definición simbólica del migrante}

Las distintas modalidades de residencia de los migrantes han adquirido relevancia como fenómeno en sí y, sobre todo, si se contextualiza en los límites propios de un migrante que arriba a un país distinto al de origen. Particularmente, si consideramos como base la definición de extrañeza que subyace en la realidad migrante, es decir, la diferencia entre migrante y nativo, o lo que es igual, la emergencia del extraño que carece de títulos de referencia válidos dada la demarcación de ese dentro/fuera que supone la sociedad de acogida; "es sobre todo, como diría A. Sayad, presencia no nacional en la nación" (Haba, 2002, p. 172).

En el caso de Chile, la distinción es aún más dramática si consideramos lo planteado por Stefoni (2011) cuando constata que, históricamente, el migrante ha sido definido como una amenaza o problema para la sociedad chilena. Particularmente, la autora plantea que al analizar la primera ley de migración, que data del siglo XIX, los migrantes eran considerados como indeseados si su llegada al país, por esos años, no tenía relación alguna con el plan de colonización impulsada por el Estado. En la misma línea, pero

2 Véanse Durston (2000) y Putnam (1993). 
más tarde, durante la dictadura de Pinochet, Stefoni (2011) plantea que los migrantes eran definidos como un peligro para la sociedad chilena, es decir, como un problema de seguridad que, y ya en democracia, persiste en tanto problema, pero ahora ya como un problema social.

Lo interesante es que en todas estas narrativas encontramos un punto en común, la del extranjero cuya sola presencia constituye una amenaza para el orden social, representación que encuentra terreno fértil en la reproducción de estructuras de discriminación y exclusión (Stefoni, 2011, p. 2).

Una política migratoria que a todas luces se aleja, acaso no es contraria, a facilitar las bases y procesos que permiten promocionar la inclusión de los migrantes (Cano y Soffia, 2009). Más bien, y siguiendo las palabras de Vior (2009), estamos ante un "Estado que realiza permanentemente una selección étnica y cultural discriminando simbólica y materialmente entre aptos e inhabilitados para participar en la comunidad de los ciudadanos" (p. 13).

Por tanto, y dado este complejo contexto, esto es, una definición problemática y/o criminal de los migrantes, cabe plantearse entonces, cuáles podrían ser los mecanismos que se pueden promover para facilitar la inclusión de las personas migrantes en la sociedad chilena.

Una revisión de la literatura evidencia que no son pocos los y las que plantean como solución, o el camino que la permita, una capitalización de derechos para los migrantes, en tanto mecanismo eficaz para superar estas problemáticas (De Julios, 2003; De Lucas, 2009; De Lucas et al., 2008; Roig, 2004). Por ejemplo, el acceso a derechos civiles permite superar los límites de: a) la lógica estado-nación que define la diferencia y jerarquización entre nacionales y migrantes (límite político); b) la dicotomía entre la cultura de origen y la de acogida (límite cultural); y c) la distribución diferencial de los recursos materiales entre nativos e inmigrantes (límite económico-laboral). Por tanto, es en el proceso de superación de dichos límites cuando los inmigrantes desarrollan y alcanzan mayores niveles de inclusión social en la sociedad de acogida (Albite, 2005; García, 2008; Jensen y Valdebenito, 2010 y Thayer, 2013).

Para el caso chileno, aunque escasas, es posible encontrar algunas referencias respecto de la capitalización de derechos. Por ejemplo, para Stefoni (2011), la actual gestión de la migración se ha basado en implementar programas sociales puntuales que facilitan el 
acceso de los migrantes a los sistemas de protección social y, desde ello, asegurar derechos que se han vulnerado desde largo tiempo.

\section{Nacionalidad y residencia: una capitalización formal legal equivalente, pero socialmente diferenciada}

Los diferenciados stocks de capital formal legal se correlacionan, a su vez, con distintas figuras administrativas-legales de residencia, y que varían según el país en el cual la persona decida asentarse. Así, por ejemplo, a nivel latinoamericano la realidad es diversa y se expresa en un continuo que va desde países que prohíben toda participación política de los migrantes extranjeros en México, Nicaragua y República Dominicana, pasando por la prohibición solo en procesos electorales de carácter nacional en Argentina y Costa Rica, hasta países como Chile o Uruguay que reconocen todos o casi todos los derechos de participación política (participación cívica, electoral, asociativa, etc.) luego de cumplir variados requisitos de residencia (Cernadas, 2011).

Para el caso específico de Chile, la estadía de un sujeto migrante resulta compleja y puede implicar tener diversos status de residencia, desde una residencia temporal hasta la naturalización y sus correspondientes derechos de ciudadanía. Pero la nacionalidad y ciudadanía son solo algunos de los estadios que puede alcanzar un migrante, quizás los más complejos y difíciles. En cualquier caso, para todo migrante los estadios de residencia emergen como el primer límite entre la irregularidad y la estancia legal, a la vez que una de las primeras etapas, necesarias aunque no suficientes, que todo migrante extranjero debe superar para alcanzar, si quiere, el estadio de mayor capitalización: la nacionalidad por adquisición luego de residir, como mínimo, por más de cinco años en el país (Stefoni, 2004).

Para el caso migrante, los diferentes estadios de residencia determinan, casi de forma creciente, las opciones para superar los límites que su propia condición de migrante impone. En el caso de la residencia temporaria (en la mayoría de sus tipos), las

posibilidades de desarrollo de los migrantes son más bien escasas toda vez que la actual normativa posiciona a los migrantes que se acojan a este beneficio como personas especiales que deben cumplir diversos requisitos para ejercer alguna actividad laboral en el país. Tal es el caso, por ejemplo, de la visa temporaria para dependientes, en tanto el migrante se define como un sujeto pasivo del titular de residencia dada la expresa prohibición para trabajar. Así, y solo después de dos años de renovar este permiso 
de residencia, el migrante puede solicitar la denominada residencia permanente o definitiva ${ }^{3}$. Lo relevante, para los términos de este escrito, radica en que este permiso definitivo abre todas las oportunidades a los migrantes para poder residir en el país, particularmente: la permanencia definitiva es el permiso otorgado a los migrantes para residir indefinidamente en Chile y desarrollar cualquier actividad lícita en el país ${ }^{4}$. Es decir, este status abre, al menos en términos legales, la frontera que se explicita en los tipos de visa temporaria, lo que supone, en el plano de derechos, evitar la perpetuación de situaciones de desigualdad (De Julios, 2003; Rinken, 2003). Más aún, la amplitud de derechos que entrega esta residencia definitiva pareciera no generar diferencias importantes con los derechos y deberes que pudiese entregar, por ejemplo, la naturalización de los sujetos, tal como lo afirma Stefoni (2004),

(...) el principio de ius domicili [...] permite entregar a los extranjeros con más de 5 años de residencia una visa de residencia definitiva, que les otorga prácticamente los mismos derechos y obligaciones que cualquier chileno, con excepción de realizar el servicio militar y postular a cargos públicos que requieran elecciones populares (Stefoni, 2004, p. 332).

En esta línea, parece sensato pensar que no es preferible iniciar un proceso de nacionalización frente a la de residencia definitiva, si lo que se busca es contar con un status que permita superar los límites de la condición migrante. Incluso, Actis (2002) plantea que la ciudadanía, desde un piso mínimo de valores democráticos, entregaría una titularidad de derechos que permitiría a los inmigrantes defender sus intereses en un plano de mayor igualdad, incluso esto podría derivar, de suyo, en un real acceso a la igualdad (Actis, 2002; Luque, 2007), algo que ya deja entrever Stefoni cuando plantea que:

Lamentablemente, y pese a que todos los países cuentan con políticas de naturalización, la sola obtención de esta membresía no asegura en ningún caso la igualdad de condiciones sociales, económicas, culturales y políticas para los inmigrantes en la sociedad de llegada (Stefoni, 2004, p. 332).

3 Según normativa publicada por el servicio de extranjería chileno: http://www.extranjeria.gob.cl/residenciatemporaria/.

4 Según normativa publicada por el servicio de extranjería chileno: http://www.extranjeria.gob.cl/permanenciadefinitiva-2/.

\section{Si Somos Americanos. Revista de Estudios Transfronterizos}


Llegados a este punto, pareciera entonces que el reclamo por la mejora en las condiciones de permanencia de los migrantes, incluso si se llega a la problematización de su integración social, se juega en la mera capitalización de derechos y mejoras de sus condiciones sociales, por lo que alcanzar una residencia definitiva sería deseable y, sobre todo, suficiente.

No obstante, lo dicho hasta aquí también genera una serie de preguntas, tanto desde la perspectiva del migrante como de la sociedad de acogida. Por ejemplo, cabe preguntarse si es posible que la sola capitalización formal legal sea necesaria y suficiente para la inserción de los migrantes en la sociedad chilena o, incluso, si esta naturalización del migrante, además de cumplir la función capitalizadora de la residencia, también genera externalidades positivas (capitalización social) para la propia sociedad de acogida.

\section{La naturaleza social de la nacionalización}

Para contestar estas preguntas debemos comenzar por considerar, tal y como lo plantea Rodriguez-Drincourt (1999), que la naturalización es, primero, un lazo jurídico basado en un hecho social de unión, más específicamente, una relación entre el migrante y la sociedad de acogida que se funda en una solidaridad efectiva y multidimensional, esto es, desde una base objetiva de intereses hasta otra de sentimientos de pertenencia, y que se expresa en una reciprocidad formal de derechos y deberes. Es decir, y tal como lo explicita Vergnaud (1995), la nacionalidad, entendida como un concepto sociológico, apunta a una persona que se une a una comunidad, lo que significa superar la concepción jurídica donde el sujeto se somete, en la verticalidad y jerarquía, al Estado en tanto componente de su población. Por tanto, bajo la construcción de la nacionalidad está la voluntad coordinada de un conjunto de sujetos (y de los propios migrantes al nacionalizarse) para definir la formación de un Estado, y que se traduce en una mentalidad particular, una forma de pensar y sentir, que se expresa en diversos ámbitos sociales (Hauriou, 1930; Rodriguez-Drincourt, 1999).

En al ámbito migrante, esta especificidad legal formal supone una realidad particular, más allá del transnacionalismo político, en donde el migrante logra una inclusión legal en la frontera de la membresía nacional y que conlleva ciertos derechos particulares, tales como, la reagrupación familiar, el libre tránsito entre los estados de origen y recepción, el voto transnacional, etc. (Bauböck, 2003); lo que finalmente explicita una membresía formal de los individuos y su sujeción al poder del Estado (Escobar, 2005), generando 
con esto una noción de pertenencia y de igualdad entre los miembros: una ciudadanía formal y una sustancial (Bertossi, 2003).

No obstante, la capitalización formal legal requiere de otros elementos necesarios para su existencia y ejercicio, particularmente, de su orientación hacia la propia sociedad de acogida, tal y como lo postula Celso Lafer (1994), cuando en su reflexión sobre el derecho a tener derecho, en tanto elemento fundante de la ciudadanía, supone su validación desde la propia convivencia colectiva y requiere, por ello, del acceso al espacio público que, en su devenir, permite la construcción de un mundo común. De igual forma, es en este sentido en el cual Touraine (1994) considera como segunda condición para la democracia que los gobernados, en su relación con el Estado, participen de la vida pública y se sientan ciudadanos, específicamente:

Esto supone ser consciente de que uno pertenece a una sociedad política [...] Ser ciudadano significa sentirse responsable del buen funcionamiento de las instituciones que respetan los derechos del hombre y permiten una representación de las ideas y de los intereses (Touraine, 1994, p. 324).

En esta línea, pero bajo la especificidad de la realidad migrante chilena, Luque (2004) plantea que la configuración de la ciudadanía de los peruanos en Chile se verifica como un proceso que se despliega en tres dimensiones. La primera, denominada como procedimental, en tanto conjunto de obligaciones y requisitos que debe cumplir en términos legales para participar del pleno derecho en la sociedad de acogida, lo que ya hemos definido como capitalización formal-legal. La segunda dimensión es aquella en donde los sujetos migrantes y nativos se homologan internamente al ser miembros de una misma comunidad con una identidad política que se diferencia de otras. Finalmente, la tercera dimensión, de tipo simbólica, hace referencia a la actividad identitaria expresada

en una cultura política y en un espacio público propio de la comunidad a la que se pertenece, y que en el caso migrante hace referencia a la sociedad de acogida.

\section{El compromiso cívico como expresión simbólica de la nacionalización}

En palabras de Noya (1997), la naturalización es fruto de una lucha por participar en la comunidad, es decir, una acción deliberada y orientada a la inclusión mediante la participación en el espacio público donde no se obvia la dimensión formal legal, en tanto 
reconocimiento de derechos y deberes, sino más bien, se entiende como un proceso de mayor complejidad que, además de la capitalización formal-legal, suma las dimensiones social y política cuando se reclama la presencia y el protagonismo activo de los migrantes en el espacio público, es decir, una incorporación a una comunidad política (León, 2008; Martínez, Tucho y García de Madariaga, 2005). En síntesis, la construcción de una ciudadanía que:

(...) no queda vinculada únicamente al principio de soberanía ni se encuentra limitada formalmente a instituciones y prácticas políticas [...] no depende de una concepción unitaria o simple del bien común. Es el resultado de diversas prácticas jurídicas, políticas y simbólicas a través de las cuales los ciudadanos acceden a bienes materiales y simbólicos en diversos ámbitos, fundamentalmente en la sociedad civil (White, 2006, p. 115, en García, 2010).

En esta línea, Luque (2007) suma un elemento central que se debe considerar a la hora de comprender el proceso de construcción de la ciudadanía de los migrantes en Chile. Específicamente, plantea que la ciudadanía es una expresión política de la naturalización, esto es, una construcción bidireccional entre el Estado y los sujetos migrados, y donde el migrante nacionalizado se define como: “(...) agencia productora de ciudadanía que va a transformar su entorno político debido a su acción afirmativa y va a constituirse paulatinamente en 'sujeto de derecho' o en "sujeto en busca de derechos y compromisos cívicos"' (Luque, 2007, p. 124).

Así, lo central surge en el segundo componente de esta ecuación, en el compromiso cívico como expresión de la ciudadanía. Un compromiso cívico que, como capitalización social, supone para Putnam (1993) una disposición a colaborar con otros con el fin de conseguir un bien público (Lechner, 2000). Más específicamente, es un compromiso que emerge por el interés en los asuntos públicos, una relevante participación electoral y, sobre todo, por la intensidad y densidad de la vida asociativa del colectivo migrante. En consecuencia, el compromiso cívico de los migrantes se manifiesta como una forma de participación activa en asuntos públicos de la sociedad de acogida dada su participación en asociaciones y organizaciones civiles en las que prevalece el encuentro y relación con otros “iguales" (Martínez et al. 2005), de ese "yo" en el "nosotros" (Fortuny y Friedmann, 2014). Tal como lo plantea León (2008):

Pero la ciudadanía universal no sólo se concibe como el derecho de todos los seres humanos a acceder a ciertos derechos considerados como fundamentales, o a la ampliación progresiva de éstos; la ciudadanía universal implica también participación, 
movilización y demanda colectiva, es decir, se trata de una ciudadanía activa (León, 2008, p. 178).

Por tanto, cuando hablamos de naturalización migrante no solo nos referimos a una definición legal-formal, sino que también a la agencia de los migrantes que se expresa en la acción participativa que despliegan en la sociedad de acogida, es decir, por su compromiso cívico. Este compromiso cívico, en tanto capital social, emerge desde su propia particularidad migrante, y que en la literatura académica ha sido definido de distintas formas, algunas son: capacidad de organización y acción ciudadana (Garay, 2009), actividades asociativas que se centran en un tema social, o causas tales como el medio ambiente, un partido político, los derechos humanos, $\mathrm{u}$ otras causas que no implican la interacción directa con los necesitados (Pérez et al., 2010), asociacionismo y acción política (Guía, 2014), coaliciones cívicas de los inmigrantes en los procesos de formulación de políticas (León, 2008). En cualquier caso, y a pesar de la diversidad conceptual, buena parte de estas definiciones conllevan un claro denominador común que dice relación con la participación asociativa de los propios migrantes, un elemento que para el caso de esta investigación toma forma y operacionalización tal y como la OCDE define el compromiso cívico en su informe sobre nacionalidad e integración migrante (2015): "There are a number of indicators that could be used to measure civic commitment. Examples include: membership of community associations, sporting or leisure activities, neighbourhood committees, trade unions, political parties, and volunteer activities" (OCDE, 2015, p. 208).

\section{Metodología}

\section{Método de estudio}

Este trabajo tiene como objetivo indagar sobre la expresión de la dimensión simbólica de la nacionalidad en los migrantes naturalizados en tanto stock de compromiso cívico y, más específicamente, bajo la forma de participación asociativa. Para esto, se plantea evaluar como hipótesis si los naturalizados chilenos evidencian un mayor stock de participación asociativa respecto de los no nacionalizados chilenos. Aún más, también se busca probar 
la capacidad explicativa que tiene la variable nacionalización sobre los distintos niveles de participación asociativa de los migrantes.

\section{Fuente de casos}

Evaluar la relevancia de la naturalización de los sujetos migrantes resulta complejo en términos metodológicos porque son escasos los instrumentos o estudios que consideran muestras de alcance representativo sobre la población migrante en Chile. No obstante, y luego de realizar variados procesos de exploración e identificación, se considera pertinente explotar los datos aportados por la encuesta CASEN 2015. Una encuesta diseñada e implementada por el Ministerio de Desarrollo Social bajo un muestreo probabilístico de cobertura nacional, lo que permite y facilita, en definitiva, el análisis de la población migrante en Chile. Además, y a falta de datos censales actualizados sobre la población, es esta encuesta la que se ha legitimado por parte de diversos actores, gubernamentales y académicos principalmente, para cuantificar y caracterizar la población residente y ciudadana del país.

\section{Selección de casos}

Se consideró utilizar una submuestra de la encuesta CASEN 2015. Particularmente, se analizaron los casos donde los respondientes cumplían con dos condiciones:

1. Identificar el lugar de nacimiento respondiendo a la pregunta r1b (cuando usted nació, ¿en qué comuna o país vivía su madre?), pudiendo identificar a los nativos o los que nacieron en Chile; o migrantes si nacieron en otro país 5 .

2. A partir de esto, se caracterizó a los respondientes en función de su status de residencia, esto es, utilizando la pregunta rla (¿cuál es la nacionalidad del integrante?) y donde se consideraron las tres alternativas de respuesta: 1) chilena (exclusivamente), 2) chilena y otra, y 3) otra. Así, los entrevistados nacidos en el exterior y que, a la vez, poseen una nacionalidad única distinta a la chilena, se clasificaron como migrantes

5 La modalidad de migración que captan el censo y la CASEN es la denominada "migración de toda la vida" o "absoluta", que se captura cotejando región de residencia habitual con la región de nacimiento (Vignoli, 2008). 
extranjeros; por otro lado, los migrantes (nacidos en el exterior) y que poseían la nacionalidad chilena y otra, ambas a la vez, evidencia la combinatoria a partir de la cual se definió al subconjunto de los migrantes nacionalizados.

Si bien esta caracterización define claramente los grupos a comparar, es necesario, todavía, controlar el efecto de dos variables que pudiesen interferir en el análisis de los datos. Para ello, se seleccionaron personas mayores de 18 años, esto porque los menores de edad tienen, en términos legales, menos autonomía para desarrollar actividades que la ley limita, por ejemplo, asociarse a organizaciones sociales. Del mismo modo, también se controló la variable tiempo de residencia en la ciudad, esto por dos razones: a) para diferenciar la migración antigua (más de cinco años de residencia) de la reciente y sus repercusiones en el arraigo comunitario (Vignoli, 2008) y b) para diferenciar a los migrantes que hayan cumplido con los cinco años necesarios para acceder a la naturalización (Stefoni, 2004). Por tanto, y a partir de la pregunta r1.c (¿En qué año llegó usted al país?), se consideraron en el análisis solo los casos en que los migrantes llevasen, como mínimo, cinco años de residencia en Chile.

\section{Análisis de los resultados}

Si ya es difícil acceder a datos fiables sobre migrantes en Chile, el análisis de estos en función de variables como la nacionalización resulta, a ratos, aún más complejo y difícil, dado que muy rara vez se pregunta esta variable de forma directa en las encuestas de alcance nacional. En este contexto, una de las aproximaciones que mejor nos puede dar una idea introductoria sobre los migrantes nacionalizados lo entrega la propia Dirección de Extranjería y Migración (DEM), a través de la figura 1, en donde se puede observar la

tendencia, desde el año 2006 hasta el 2014, del número de migrantes que han adquirido la nacionalidad chilena y que, en total, para este periodo, sobrepasan los 7.800 casos. 


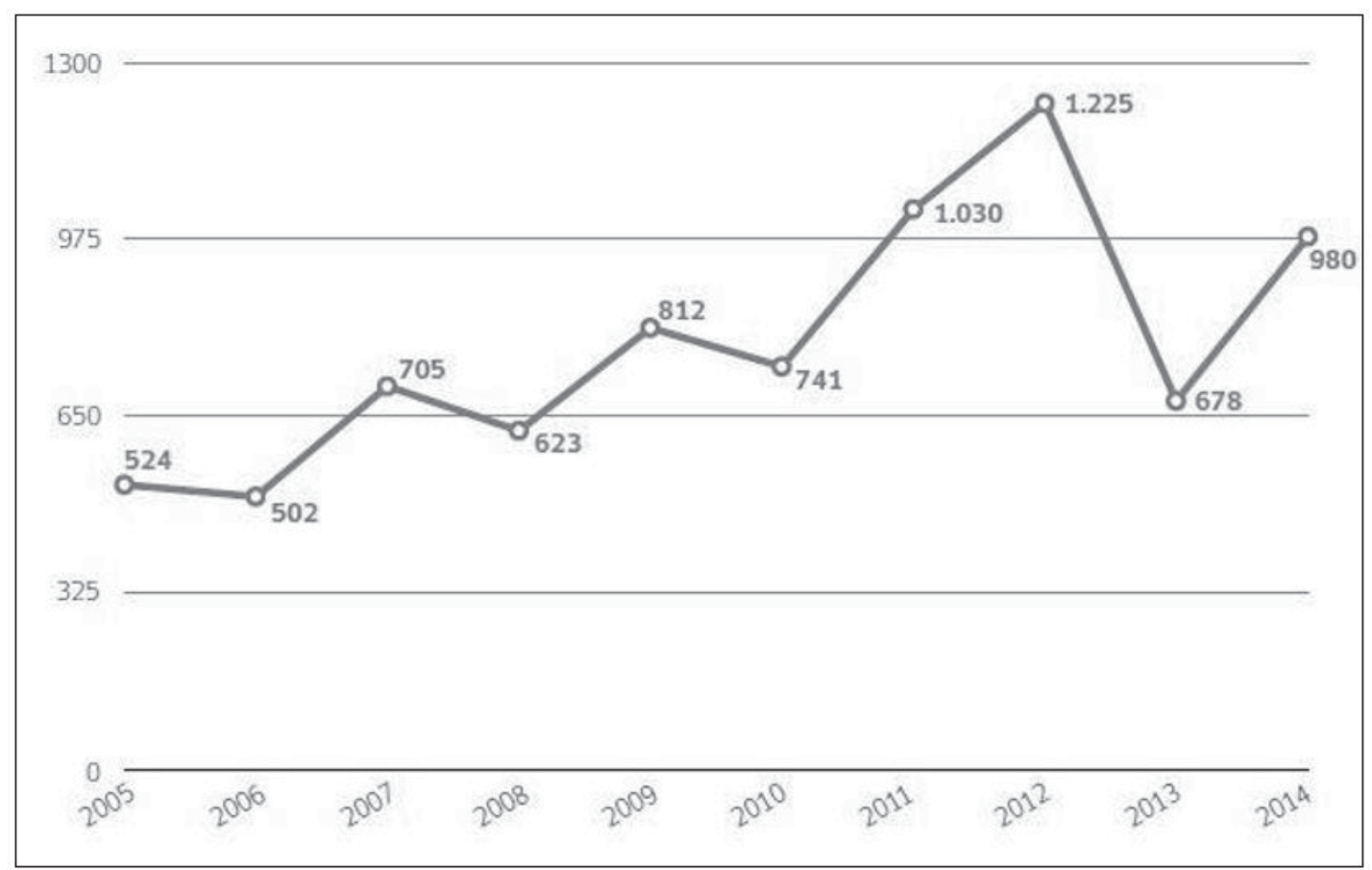

Figura 1. Número de migrantes nacionalizados entre los años 2005-2014. Adaptado de Dirección de Extranjería y Migración (DEM, 2016) .

Una lectura del número de naturalizaciones a través de los años revela, primero, cómo la nacionalización es en sí un fenómeno que irrumpe con fuerza creciente en los últimos años y, segundo, entrega una perspectiva longitudinal de los casos que precisamente trabajamos en esta investigación, particularmente sobre migrantes con más de cinco años de residencia.

\section{Nacionalización inmigrante y participación en organizaciones o grupo organizados: una capitalización social}

Para responder a nuestra hipótesis, se hace necesario efectuar una primera aproximación a los niveles de participación de los encuestados en organizaciones sociales de la sociedad chilena, según sean nativos chilenos o migrantes y, en esta última categoría, evaluar las diferencias de participación entre los subgrupos migrantes nacionalizados y no

6 Departamento de Extranjería y Migración, Anuario Estadístico Nacional 2005-2014, Santiago de Chile, 2016. 
nacionalizados. Para esto se presenta la figura 2, que presenta las proporciones de nativos y migrantes según su nivel de participación en organizaciones o grupo organizado en los últimos doce meses $^{7}$ (CASEN, 2015).

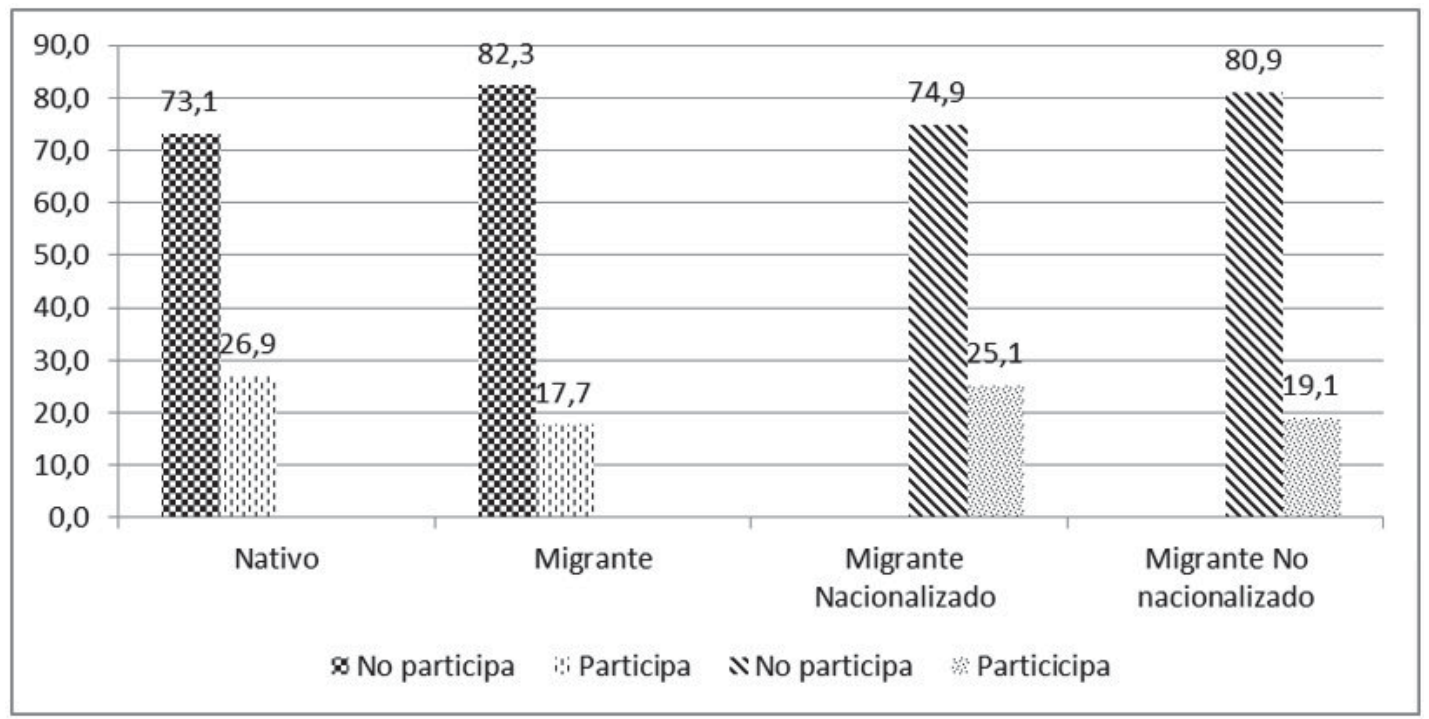

Figura 2. Distribución de la población nacida en Chile e inmigrantes mayores de 18 y entre migrantes nacionalizados y no nacionalizados con más de cinco años de residencia años según niveles de participación en organizaciones sociales (\%). Fuente: elaboración propia a partir de la CASEN (2015).

Una inspección de la primera parte de la figura 2 (sector izquierdo de la figura: Nativo y migrante) evidencia que el nivel de participación, para ambos grupos, no supera el $30 \%$. En el caso de población nacional se evidencia que algo menos del $27 \%$ participa en organizaciones o grupo organizados de la sociedad civil. Por el otro lado, en el caso del colectivo migrante, esta razón no llega al 18\%. Ahora bien, si analizamos las distribuciones de participación al interior del grupo migrante, vemos que se evidencian diferencias relevantes en los niveles de participación según sean estos migrantes nacionalizados o no; particularmente, los primeros alcanzan una proporción algo superior al 25\%, mientras que los segundos no alcanzan siquiera el 20\%. Por tanto, si bien los migrantes en general participan menos que los nativos, al interior de población migrante el grupo

7 Pregunta r6: En los últimos 12 meses, ¿ha participado en alguna de las siguientes organizaciones o grupo organizado? (Mostrar tarjeta r6 organizaciones sociales - Lea alternativas - Registre la más importante) (CASEN, 2015). 
de nacionalizados resulta ser el más participativo, incluso son quienes concentran proporciones similares a las presentadas por los nativos.

Ahora bien, y considerando que el objetivo de este trabajo es evidenciar la relación de dependencia entre dos variables: nacionalidad y compromiso cívico, en tanto la segunda como expresión simbólica de la primera, es que utilizaremos diversas pruebas estadísticas, particularmente nos referimos a la prueba de independencia de chi-cuadrado, a la prueba de magnitud y significancia de la relación de $\mathrm{V}$ de Cramer y al análisis de los residuos tipificados corregidos (RTC). Esta esta última prueba permite evaluar la direccionalidad de la relación entre variables cualitativas ${ }^{8}$. Cabe precisar que para todas estas pruebas se asume, como hipótesis nula, que existe independencia entre las variables, es decir, la distribución de los casos, según la combinatoria entre las categorías de ambas variables, no revela una distribución fruto de la dependencia entre ambas.

\section{Nacionalidad y participación en organizaciones sociales}

En función de lo anterior, se considera especificar el análisis, ahora solo para el grupo migrante según su status de naturalización y los años que contabilizan residiendo en el país; además, se procedió a la recodificación de categorías de la pregunta "r6" a fin de alcanzar un número de casos suficientes que permitiera realizar la mayor cantidad de comparaciones de contingencia. Hecho esto, un primer análisis de la tabla 1 arroja evidencia sobre la relación de dependencia entre las variables nacionalización y participación en organizaciones sociales. Específicamente, se constata que la proporción de migrantes nacionalizados que participa en organizaciones sociales $(25,1 \%)$ obtiene un diferencial a su favor de un $6 \%$ respecto de la proporción de migrantes no nacionalizados que también participan en dichas organizaciones (19,1\%), una diferencia que, a la luz de los resultados de la prueba de chi-cuadrado, resulta estadísticamente significativa $\left[\chi^{2}(6\right.$, $\mathrm{N}=201105)=3295,604, \mathrm{p}<.05)]$.

En esta misma línea, y según los resultados arrojados por la prueba V de Cramer (.13, $\mathrm{p}<.05$ ), se puede confirmar, primero, que esta relación de dependencia (estadísticamente significativa) no se encuentra distorsionada por el tamaño de la muestrea analizada $\mathrm{y}$, segundo, que la relación posee una fuerza considerable que también resulta ser estadísticamente significativa, es decir, entre ambas variables existe una dependencia que puede ser definida como fuerte y significativa.

$8 \quad$ Véase Siegel, S. y Castellan, J. (2012). 


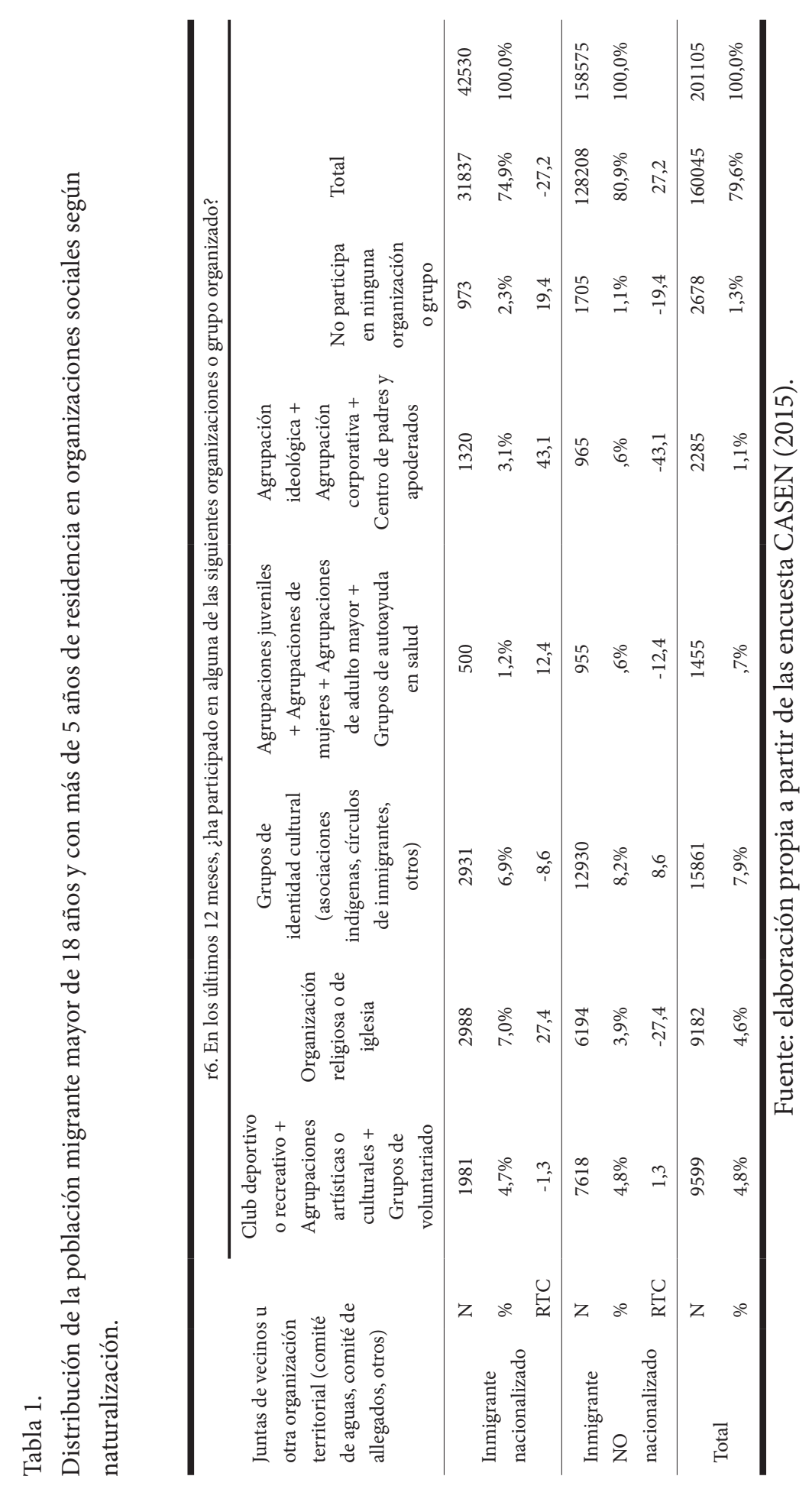

142 Si Somos Americanos. Revista de Estudios Transfronterizos 
Un segundo elemento que requiere un análisis detallado dice relación con identificar los tipos de organizaciones en las cuales los migrantes participan. Para esto, y según la inspección de los residuos tipificados corregidos (RTC), se constata que los migrantes nacionalizados concentran su actuar en dos tipos de asociaciones bastante particulares: las primeras, que pueden ser denominadas como las organizaciones recreativas y generalistas, concentran un 7\% de las menciones y que, en términos comparados con los no nacionalizados, evidencian una diferencia de 3,1 puntos porcentuales a su favor, una diferencia que resulta ser estadísticamente significativa. Por otro lado, la participación en organizaciones de tipo "religiosa o de iglesia" registra un porcentaje similar (6,9\%), pero en este caso, dada su comparativa con los no nacionalizados, se evidencia una diferencia negativa de $1,3 \%$, lo que resulta estadísticamente significativa a favor de estos últimos. Por tanto, esto supone que para los nacionalizados este tipo de participación no resulta atractiva en términos comparados, a diferencia de lo que ocurre con las organizaciones artísticas, recreativas y de voluntariado.

Un tercer grupo de asociaciones, las que podrían ser denominadas como territoriales locales (Juntas de vecinos u otra organización territorial: comité de aguas, comité de allegados, otros), si bien concentran casi un 5\% de la nominaciones de los migrantes nacionalizados no logran, en términos comparados con los no nacionalizados, una diferencia que sea estadísticamente significativa, por lo que no se puede afirmar que alguno de los grupos defina preferencias participativas diferenciadoras respecto del otro.

Si bien el resto de categorías de organizaciones evidencian, en términos nominales, bajas frecuencias de participación, igualmente, y desde el punto de vista comparado, se evidencia nuevamente que son los nacionalizados los que alcanzan diferencias de participación a su favor que resultan estadísticamente significativas cuando se les compara con los no nacionalizados.

No obstante lo anterior, cabe destacar que para los nacionalizados las organizaciones definidas y agrupadas como Grupos de identidad cultural (asociaciones indígenas, círculos de inmigrantes, otros) superan, por muy poco, el 1\% de las elecciones. Incluso, su frecuencia es casi doblada por la siguiente categoría minoritaria: Agrupación ideológica + Agrupación corporativa + Centro de padres y apoderados, que alcanza un $2,3 \%$. Estas diferencias intragrupo e intercategorías no se observan de la misma forma cuando se analiza el grupo de los no nacionalizados, en tanto, y en la misma comparativa, se evidencian proporciones muy similares: 0,6\% y 1,1\% al comparar entre Grupos de identidad cultural y Agrupación ideológica + Agrupación corporativa + Centro de padres y apoderados, y ninguna diferencia con la categoría Agrupaciones juveniles + 
Agrupaciones de mujeres + Agrupaciones de adulto mayor + Grupos de autoayuda en salud, en donde las proporciones son idénticas.

\section{Nacionalidad y participación en organizaciones laborales y profesionales}

En continuidad con el análisis por tipo de organizaciones, el análisis se centra ahora en el nivel de participación específica de los migrantes en organizaciones laborales y profesionales. Esta línea de análisis se fundamenta en lo planteado por Aleksynska (2011), en cuanto a que el nivel de afiliación sindical es un indicador básico de la participación migrante, más aún, la participación sindical de los migrantes es la base de su participación política en la sociedad de acogida, o como lo define Martiniello (2007):

La presencia de inmigrantes en los sindicatos es un fenómeno antiguo y bien conocido en los viejos países europeos de inmigración. Ser activo en un sindicato, ya sea como partidario y miembro o como activista o en el ejecutivo, es un indicador relevante de la participación política (Martiniello, 2007, p. 17).

Además de esto, la pregunta utilizada para este análisis es distinta de la anterior, con esto intentamos triangular la información a fin de poder contrastar la validez de los resultados obtenidos. Nos referimos, particularmente, a la pregunta o24 de la encuesta CASEN (2015)9. Un detalle de los resultados se muestra, a continuación, en la tabla 2.

9 Pregunta 024: ¿En su empleo principal, pertenece a alguna de las siguientes organizaciones en relación con su trabajo? (Sindicato de empresa, inter-empresa, de trabajadores eventuales o transitorios, o de trabajadores independientes; Asociación de funcionarios; Asociación gremial sectorial y Colegio profesional. (Responda para cada organización).

\section{Si Somos Americanos. Revista de Estudios Transfronterizos}


Tabla 2.

Distribución de la población migrante mayor de 18 años y con más de 5 años de residencia en organizaciones laborales y profesionales

\begin{tabular}{|c|c|c|c|c|c|c|}
\hline & & \multicolumn{5}{|c|}{$\begin{array}{l}\text { o24. ¿En su empleo principal, pertenece a alguna de las siguientes } \\
\text { organizaciones en relación con su trabajo? }\end{array}$} \\
\hline & & $\begin{array}{l}\text { Sindicato } \\
\quad(\text { Sí) }\end{array}$ & $\begin{array}{l}\text { Asociación } \\
\text { de } \\
\text { funcionarios } \\
\text { (Sí) }\end{array}$ & $\begin{array}{l}\text { Asociación } \\
\text { gremial } \\
\text { sectorial (Sí) }\end{array}$ & $\begin{array}{l}\text { Colegio } \\
\text { profesional } \\
(\text { Sí) }\end{array}$ & Total \\
\hline \multirow{3}{*}{$\begin{array}{l}\text { Inmigrante } \\
\text { nacionalizado }\end{array}$} & $\mathrm{N}$ & 3219 & 448 & 74 & 390 & 4131 \\
\hline & $\%$ & $10,5 \%$ & $1,5 \%$ & $0,2 \%$ & $1,3 \%$ & $13,5 \%$ \\
\hline & RTC & 56,0 & 25,0 & 3,6 & 23,1 & \\
\hline \multirow{3}{*}{$\begin{array}{l}\text { Inmigrante NO } \\
\text { nacionalizado }\end{array}$} & $\mathrm{N}$ & 3680 & 363 & 180 & 320 & 4543 \\
\hline & $\%$ & $3,0 \%$ &, $3 \%$ & $0,1 \%$ &, $3 \%$ & $3,7 \%$ \\
\hline & RTC & $-56,0$ & $-25,0$ & $-3,6$ & $-23,1$ & \\
\hline \multirow{2}{*}{ Total } & $\mathrm{N}$ & 6899 & 811 & 254 & 710 & 8674 \\
\hline & $\%$ & $4,5 \%$ & $0,5 \%$ & $0,2 \%$ &, $5 \%$ & $5,7 \%$ \\
\hline \multirow{4}{*}{ Estadísticos } & $\begin{array}{l}\text { Chi - } 2 \\
\text { /gl }\end{array}$ & $3132,461 / 1$ & $627,070 / 1$ & 12,825 & $533,814 / 1$ & \\
\hline & Sig. & 0,000 & 0,000 & 0,000 & 0,000 & \\
\hline & $\begin{array}{l}\text { V de } \\
\text { Cramer }\end{array}$ & 0,144 & 0,064 & 0,009 & 0,059 & \\
\hline & Sig. & 0,000 & 0,000 & 0,000 & 0,000 & \\
\hline
\end{tabular}

Fuente: elaboración propia a partir de las encuesta CASEN (2015).

Tal como se puede observar en la tabla 2, la comparativa entre migrantes nacionalizados y no nacionalizados evidencia, para todos los tipos de participación, diferencias a favor de los primeros respecto de los segundos. Sin embargo, la única diferencia que resulta estadísticamente significativa, siempre a favor de los nacionalizados, es la que se refiere a su participación en los sindicatos, ya que es la única distribución que obtiene una puntuación mayor a 0,10 en la prueba de $\mathrm{V}$ de Cramer. Por el contrario, el resto de diferencias resultan relevantes solo a nivel descriptivo. En consecuencia, es posible afirmar, con cierta garantía estadística, que la participación de los migrantes en las 
organizaciones sociales y sindicales tiene una relación de dependencia con el status de residencia que estos tengan, particularmente si se encuentran nacionalizados.

\section{Nacionalidad y factores sociodemográficos explicativos del compromiso cívico}

En este apartado se pretende evaluar en qué medida el compromiso cívico, entendido como la participación en organizaciones sociales, o más específicamente, qué probabilidad de participar en estas organizaciones sociales se explica de forma significativa por parte de la variable status de nacionalidad pero, esta vez, controlando el efecto de otras variables que, según la literatura, pueden tener cierto efecto en dicho comportamiento asociativo (de los Mozos, 2013). Para ello, evaluamos la pregunta r6 (En los últimos 12 meses, ¿ha participado en alguna de las siguientes organizaciones o grupo organizado?) como variable dependiente, recodificada en dos categorías: participa y no participa. Además, se consideró como variables independientes el status de nacionalidad (nacionalizado y no nacionalizado) junto a las variables sociodemográficas: ocupación (asalariado, patrón/ cuenta propia y familiar no remunerado, inactivo o desocupado), situación de pobreza multidimensional (pobre/no pobre), sexo (hombre/mujer), años de escolaridad, edad y años de llegada al país. De esta forma, y mediante el análisis de regresión logística, los resultados de los efectos para los distintos modelos se presentan en la tabla 3 .

Tal como se puede observar, los estadísticos definidos alcanzan porcentajes de estimación bastante modestos para los modelos analizados. No obstante ello, nuestro objetivo no es la construcción de un modelo explicativo que permita predecir el comportamiento de la variable dependiente definida como participación en organizaciones y grupos sociales; más bien, el objetivo es evaluar si el status de nacionalidad de los migrantes se relaciona con la probabilidad de participar en las organizaciones más allá de una dependencia correlacional y, si esta relación es significativa cuando intervienen otras variables en la interacción. En ello, y según los datos revelados, podemos confirmar que esta relación es consistente y alcanza, nuevamente, una relevancia estadísticamente significativa, particularmente, podemos afirmar que los migrantes nacionalizados tienen una mayor probabilidad de participar en organizaciones sociales (1.067 veces más) que los migrantes no nacionalizados, siempre y cuando el resto de variables independientes se mantengan constantes.

146 Si Somos Americanos. Revista de Estudios Transfronterizos 


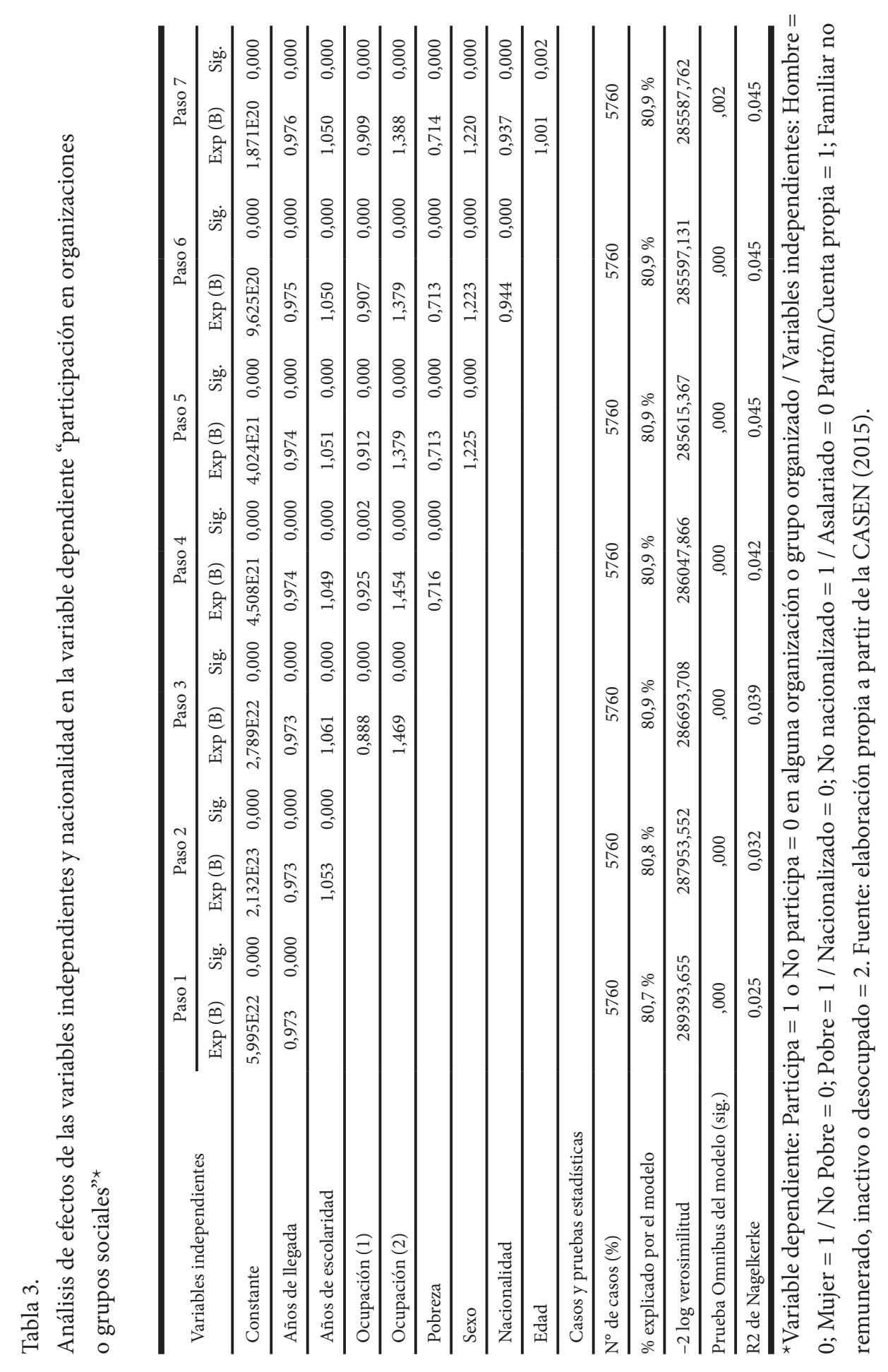


Además de lo anterior, cabe mencionar que el método utilizado para el análisis de regresión (condicional hacia adelante) solo va integrando variables independientes al modelo siempre y cuando aporten de forma significativa a la capacidad explicativa del modelo, de lo contrario, son excluidas. En este sentido, y analizando el lugar de entrada de la variable nacionalidad en el modelo, podemos identificar que, antes de ella, hay otras variables con mayor relevancia a la hora de explicar la probabilidad de participar en organizaciones o grupos sociales (Años de llegada, Años de escolaridad, Ocupación, Pobreza y Sexo). No obstante, lo relevante, dado el propósito de este escrito, es que la variable status de nacionalidad igualmente es incorporada al modelo, por lo que revela un aporte significativo y un efecto sobre la participación, lo que, finalmente, nos lleva a reafirmar la relación de dependencia con la variable compromiso cívico; incluso, cuando en esta evaluación se controla la relevancia de otras variables predictoras del comportamiento asociativo de los migrantes.

\section{Conclusiones y discusión}

A partir del análisis presentado, es posible concluir que el compromiso cívico de los inmigrantes, al menos para el caso chileno y según los datos entregados por la CASEN 2015, está relacionado con el status de naturalización, particularmente un compromiso analizado y operacionlizado desde la participación en grupos y organizaciones sociales $y$, también, desde la participación en organizaciones de tipo laboral y profesional, particularmente los sindicatos. Incluso, respecto de la participación en las institutions of mainstream societies, la nacionalidad puede ser considerada como un factor explicativo a la hora de evaluar la probabilidad de participar en dichas organizaciones sociales. Elementos todos que son concordantes con diversos informes y artículos relativos a esta misma cuestión (Biezeveld y Entzinger, 2003; Huddleston, Niessen y Tjaden, 2013; OCDE, 2015; Pajnik y Bajt, 2013; Terriquez y Kwon, 2015; ; Van Londen et al., 2007; Vogel y Von Ossietzky, 2005).

$\mathrm{Y}$ es que parece totalmente plausible concluir que la dimensión simbólica de la naturalización migrante se expresa de forma clara y contundente en el mundo asociativo migrante. Así, si bien la culturalización del debate migratorio tiene aspectos positivos, la prioridad se halla en la política, puesto que si no hay participación política de los inmigrantes en la sociedad de acogida, no hay sentimiento de pertenencia, ni conciencia de identidad, y difícilmente cabe hablar de inclusión social de los migrantes (De Lucas, 2009; De Julios, 2003). Algo que para la realidad europea se evidencia con mediana 
claridad, si consideramos las conclusiones que plantean Huddleston et al. (2013), en cuanto a que los migrantes naturalizados conllevan una externalidad positiva de la ciudadanía dado que mejoran sus formas de integración política económica y social, independiente de si las políticas migratorias de la sociedad de acogida son más o menos restrictivas. Por otro lado, los migrantes que llevan más de diez años de residencia, pero aún no se han naturalizado, continúan siendo vistos como extranjeros y, en gran medida, son recurrentemente excluidos del proceso democrático.

En consecuencia, la naturalización puede reducir las barreras legales reales (o percibidas) tanto para facilitar su acceso al mercado del trabajo como la participación social. Y por parte del migrante, es una señal de que se quiere establecer a largo plazo y participar de la sociedad de acogida. Pero no es solo la mayor participación en general o global lo que supone una capitalización social del sujeto migrante nacionalizado con miras a favorecer su inclusión social, sino que también la dirección de dicha participación. Y es que una tendencia mayoritaria a participar más en organizaciones de la sociedad de acogida complementada con una orientación menor a participar en asociaciones propias de los colectivos migrantes favorece, de por sí, su relación con la sociedad de acogida, facilita una mayor interacción con los nativos y supone, siguiendo modelo de integración de Berry (2002 y 2006), dar cuenta de uno de los elementos basales que requiere todo procesos de integración social, esto es, la interacción y relación entre los extraños y los originarios. Por tanto, la tendencia exogámica de los migrantes nacionalizados supone un mecanismo de integración claro y contrario a procesos de clausura endogámica que suponen una alta y exclusiva participación en organizaciones migrantes.

No obstante, esta exogamia participativa no solo facilita su integración, también genera externalidades positivas para la propia sociedad de acogida, particularmente, una capitalización social que se orienta a una participación activa en la sociedad civil chilena. Y es que si se consideran diversos y sendos estudios que hablan de la cada vez más paupérrima y creciente desafección política y/o baja participación asociativa de los nativos chilenos (Montecinos, 2014; Reyes, 2014; Rengifo, 2008; Jiménez y Sánchez, 2008), se deduce, entonces, que los migrantes nacionalizados suponen un aire fresco a esta realidad poco alentadora que vive la sociedad civil chilena.

En definitiva, los migrantes naturalizados emergen como nuevos actores claves y relevantes para dinamizar las organizaciones de la sociedad civil chilena, y ponen en jaque y neutralizan esa idea de que la nacionalización migrante, en tanto mera capitalización formal legal, podría ser considerada como un favor que se le hace al migrante (Stefoni, 
2011), cuando en realidad pareciera que la nacionalización de los migrantes supone o termina siendo más bien un aporte para la propia y alicaída sociedad civil chilena.

A modo de conclusión general, y considerando lo anteriormente expuesto, se deduce que la expresión de la dimensión simbólica de la nacionalización conlleva una capitalización social que es complementaria de la legal, lo que supone que esta supuesta equiparación entre derechos de ciudadanía y nacionalidad, dada la similitud de derechos en los cuales capitaliza a los sujetos, es más bien inexacta y con un diferencial positivo en favor de la nacionalidad. Por tanto, la nacionalidad emerge como un mecanismo de inclusión mucho más potente y completo que la mera habilitación legal de los migrantes. Esto no quiere decir, bajo ningún modo, que la ciudadanía sea considerada como un mecanismo no deseado, más bien, es necesario ponerlo en un continuo, como un primer escalón dentro del proceso de inclusión, pero no es suficiente, ya que su no realización genera una pérdida personal para los migrantes y también para la sociedad, dada la capitalización social con la que la nacionalidad se asocia.

\section{Referencias}

Actis, W. (2002). Extranjería, nacionalidad, ciudadanía. En M. Delgado, W. Actis, D. Martucelli, I. Palacín y P. Sáez (Eds.), Exclusión social y diversidad cultural (pp. 25-37). Madrid: Tercera Prensa.

Albite, P. (2005). Inmigración y multiculturalidad: debates teóricos sobre prácticas y modelos para la convivencia. En M. Hernández y A. Pedreño (Coords.), La condición inmigrante: exploraciones e investigaciones desde la región de Murcia (pp. 211-226). Murcia: EDITUM.

Aleksynska, M. (2011). Civic participation of immigrants in Europe: Assimilation, origin, and destination country effects. European Journal of Political Economy, 27 (3), 566-585.

Bauböck, R. (2003). Towards a political theory of migrant transnationalism. International migration review, 37 (3), 700-723.

Berry, J, Phinney, J., Sam, D. y Vedder, P. (2006). Immigrant youth in cultural transition: Acculturation, identity, and adaptation across national contexts. New Jersey: Psychology Press.

150 Si Somos Americanos. Revista de Estudios Transfronterizos 
Berry, J., Marshall, S. y Cigdem, K. (2002). Handbook of Cross-cultural Psychology: Social behavior and applications. Cambridge: Cambridge University Press.

Bertossi, C. (2003). La ciudadanía francesa: debates límites y perspectivas. Revista de Occidente, 268 (5), 82-102.

Biezeveld, R. y Entzinger, H. (2003). Benchmarking in immigrant integration. Rotterdam: Report for the European Commission.

Cano, V. y Soffia, M. (2009). Los estudios sobre migración internacional en Chile: apuntes y comentarios para una agenda de investigación actualizada. Papeles de población, 15 (61), 129-167.

Cernadas, P. (2011). Luces y sombras en la legislación migratoria latinoamericana. Nueva sociedad, (233), 68-86.

Durston, J. (2000). ¿Qué es el capital social comunitario? Santiago, Chile: Naciones Unidas Comisión/CEPAL.

De Julios Campuzano, A. (2003). La paradoja de la ciudadanía: Inmigración y derechos en un mundo globalizado. Cuadernos electrónicos de filosofía del derecho, 7 (7), 1-24.

De Los Mozos, F. (2013). Capital social de las Asociaciones de Inmigrantes: asociaciones bolivianas, colombianas, ecuatorianas y peruanas en Barcelona, Bilbao, Madrid y Valencia. Bilbao: Universidad de Deusto.

De Lucas Martín, F. (2009). Inmigración, diversidad cultural, reconocimiento político. Papers: revista de sociología, (94), 11-27.

De Lucas Martín, F., Roig, M., Saura, Á., Añón, J., Mestre, R., Bergón, P. y Pérez, F. (2008). Los derechos de participación como elemento de integración de los inmigrantes. Bilbao: Rubes Editorial.

Escobar, C. (2005). La doble ciudadanía y la participación política: inmigrantes en la interacción de la política de Estados Unidos y Colombia. Análisis Político, 18 (53), $3-21$.

Fortuny, P. y Friedmann, M. (2014). Cooperation and Conflict: Catholic Churches and Latino Immigrants. Desacatos, (46), 70-87. 
Garay, A. (2009). Transnacional social mexicana Alcances y limitaciones del Programa

3x1 para Migrantes en Guerrero. Trayectorias, 11 (29), 117-131.

García, I. (2008). Herederos de la condición inmigrante: adolescentes y jóvenes en familias madrileñas de origen extranjero (Tesis Doctoral). Universidad Nacional de Educación a Distancia (UNED), Madrid, España.

García, R. (2010). Compromiso cívico y democracia: los efectos democráticos del asociacionismo sociopolítico en España. Andalucía: Centro de Estudios Andaluces.

Guía, A. (2014). The Muslim Struggle for Civil Rights in Spain Promoting Democracy through Migrant Engagement, 1985-2010. Sussex: Sussex Academic Press.

Haba, J. (2002). Trabajadores inmigrantes y acción colectiva. Papers: revista de sociología, 66, 155-186.

Hauriou, M. (1930). Précis elemental de droit constitutionnet. París: Recueil Sirey.

Huddleston, T., Niessen, J. y Tjaden, J. D. (2013). Using EU indicators of immigrant integration. Final Report for Directorate-General for Home Affairs. Bruselas: European Commission.

Jensen, F. y Valdebenito, X. (2010). De inclusiones y exclusiones: una perspectiva de la inmigración desde la Asociatividad en Chile. Miradas en Movimiento, (3), 6-38.

Jiménez, S. y Sánchez, R. (2008). La participación política de la juventud chilena. Entre las pautas clásicas y el desapego a sus instituciones. Revista observatorio de juventud, 5 (19), 18-31.

Lafer, C. (1994). La reconstrucción de los derechos humanos: un diálogo con el pensamiento de Hannah Arendt. México, D.F.: Fondo de Cultura Económica.

Lechner, N. (2000). Desafíos de un desarrollo humano: individualización y capital social. Instituciones y desarrollo, 7, 7-34.

León, M. (2008). La Ciudadanía en tiempos de migración. Hacia un periodismo cívico. Revista de Comunicación, VIII (7), 167-181.

152 Si Somos Americanos. Revista de Estudios Transfronterizos 
Luque, J. (2004). Transnacionalismo y enclave territorial étnico en la configuración de la ciudadanía de los migrantes peruanos en Santiago de Chile. Revista Enfoques: Ciencia Política y Administración Pública, 3, 81-102.

Luque, J. (2007). Asociaciones políticas de inmigrantes peruanos y la "Lima Chica" en Santiago de Chile. Migraciones internacionales, 4 (2), 121-150.

Martínez, N., Tucho, M. y García de Madariaga, J. (2005). Democracia digital: nuevos medios y participación ciudadana. Experiencias en la red de la población inmigrante en España. Portularia, $V(2), 21-34$.

Martiniello, M. (2007). Political participation, mobilisation and representation of immigrants and their offspring in Europe. En R. Baubock (Ed.), Migration and Citizenship (pp. 83--105). Ámsterdam: University Press.

Montecinos-Montecinos, E. (2014). Limitaciones del diseño institucional para una gestión municipal participativa. El caso chileno. Revista Economía, Sociedad y Territorio, VI (23).

Mora, C. (2008). The Peruvian Community in Chile. Peace Review: A Journal of Social Justice, 20 (3), 339-347.

Noya, F. (1997). Ciudadanía y capital social. Estudio preliminar en torno a Ciudadanía y clase social de T.H. Marshall. Reis, 79, 267-295.

OECD/European Union. (2015). Civic engagement of immigrants, in Indicators of Immigrant Integration 2015: Settling In. París: OECD Publishing.

Pajnik, M. y Bajt, V. (2013). Civic participation of migrant women: Employing strategies of active citizenship. En A. Floya, M. Kontos y M. Morokvasic-Müller (Eds.), Paradoxes of integration: Female migrants in Europe (pp. 97-116). Ámsterdam: Springer Netherlands.

Pérez, W., Espinoza, R., Ramos, K., Coronado, H., y Cortes, R. (2010). Civic engagement patterns of undocumented Mexican students. Journal of Hispanic Higher Education, 9 (3), 245-265.

Putnam, R. (1993). Making Democracy work. Civic Traditions In Modern Italy. New Jersey: Princeton University Press. 
Rengifo, E. (2008). Juventud y Participación política en Chile. Del ciudadano al consumidor, un tránsito forzado, versión electrónica. Revista observatorio de juventud, 5 (19), 7-17.

Reyes, P. (2014). Baja participación cívica de los jóvenes en la sociedad chilena actual. Documento de Facultad, 20 (4), 3-20.

Rinken, S. (2003). Inmigración y ciudadanía. Algunas observaciones basadas en el caso andaluz. En M. Carrasco, F. Pérez, J. Urías y Manuel Terol (Coords.), Derecho constitucional para el siglo XXI: actas del VIII Congreso Iberoamericano de Derecho Constitucional (pp. 4949-4961). Sevilla: Editorial Aranzadi.

Rodríguez, J. (2008). Dinámica sociodemográfica metropolitana y segregación residencial: ¿qué aporta la CASEN 2006? Revista de Geografía Norte Grande, (41), 81-102.

Rodriguez-Drincourt, J. (1999). La nacionalización como vía de integración de los inmigrantes extranjeros. Revista de estudios políticos, (103), 171-185.

Roig, M. J. A. (2004). Límites de la universidad: los derechos sociales de los inmigrantes. En M. Añón (Coord.), La universalidad de los derechos sociales: el reto de la inmigración (pp. 9-26). Valencia: Servei de Publicacions, Universitat de València.

Schiller, N., Basch, L. y Blanc, C. (1995). From immigrant to transmigrant: Theorizing transnational migration. Anthropological quarterly, 68 (1), 48-63.

Siegel, S. y Castellan, J. (2012). Estadística no paramétrica: Aplicada a las ciencias de la conducta. México, D.F.: Editorial Trillas.

Stefoni, C. (2004). Inmigración y ciudadanía: la formación de comunidades peruanas en Santiago y la emergencia de nuevos ciudadanos. Política, (43), 319-336.

Stefoni, C. (2011). Ley y política migratoria en Chile. La ambivalencia en la comprensión del migrante. En B. Feldman-Bianco, L. Rivera, C. Stefoni y M. Villa (Coords.), La construcción social del sujeto migrante en América Latina. Prácticas, representaciones y categorías (pp. 79-109). Quito: CLACSO Coediciones.

Terriquez, V. y Kwon, H. (2015). Intergenerational family relations, civic organisations, and the political socialisation of second-generation immigrant youth. Journal of Ethnic and Migration Studies, 41 (3), 425-447.

154 Si Somos Americanos. Revista de Estudios Transfronterizos 
Thayer, E. (2013). Expectativas de reconocimiento y estrategias de incorporación. Polis: Revista Latinoamericana, (35).

Touraine, A. (1994). Crítica de la modernidad. México, D.F.: Fondo de Cultura Económica.

Van Londen, M., Phalet, K. y Hagendoorn, L. (2007). Civic engagement and voter participation among Turkish and Moroccan minorities in Rotterdam. Journal of Ethnic and Migration Studies, 33 (8), 1201-1226.

Vergnaud, I. (1995) L 'idee de la nationalité el de la libre-disposition des peuples dans ses rapports avec Vidée de l'Etat. París: Domat-Montchrestien.

Vior, E. (2009). Participación ciudadana en las comunidades de origen inmigrante en la provincia de Río Negro. Nuevo espacio público, 2 (3), 165-198.

Vogel, D. y Von Ossietzky, C. (2005). Building Europe With New Citizens? Bruselas: European Commission. 